\title{
New technology based on clamping for high gradient radio frequency photogun
}

\author{
David Alesini, Antonio Battisti, Massimo Ferrario, Luca Foggetta, and Valerio Lollo \\ INFN-Laboratori Nazionali di Frascati, Via Enrico Fermi 40, 00044 Frascati, Rome, Italy \\ Luca Ficcadenti and Valerio Pettinacci \\ INFN-Sezione di Roma 1, Piazzale Aldo Moro 2, 00185 Roma, Italy \\ Sean Custodio, Eylene Pirez, and Pietro Musumeci \\ Department of Physics and Astronomy, UCLA, Los Angeles, California 90095, USA \\ Luigi Palumbo \\ University of Rome "La Sapienza", SBAI Department, Via Antonio Scarpa 14, 00161 Rome, Italy
} (Received 18 March 2015; published 15 September 2015)

\begin{abstract}
High gradient rf photoguns have been a key development to enable several applications of high quality electron beams. They allow the generation of beams with very high peak current and low transverse emittance, satisfying the tight demands for free-electron lasers, energy recovery linacs, Compton/Thomson sources and high-energy linear colliders. In the present paper we present the design of a new rf photogun recently developed in the framework of the SPARC_LAB photoinjector activities at the laboratories of the National Institute of Nuclear Physics in Frascati (LNF-INFN, Italy). This design implements several new features from the electromagnetic point of view and, more important, a novel technology for its realization that does not involve any brazing process. From the electromagnetic point of view the gun presents high mode separation, low peak surface electric field at the iris and minimized pulsed heating on the coupler. For the realization, we have implemented a novel fabrication design that, avoiding brazing, strongly reduces the cost, the realization time and the risk of failure. Details on the electromagnetic design, low power rf measurements and high power radiofrequency and beam tests performed at the University of California in Los Angeles (UCLA) are discussed in the paper.
\end{abstract}

DOI: 10.1103/PhysRevSTAB.18.092001

PACS numbers: 29.20.Ej, 29.25.Bx, 41.20.-q, 07.57.-c

\section{INTRODUCTION}

Photocathode rf guns are multicell standing wave (SW) structures in which the electron beam is generated by photoemission using a drive-laser pulse to illuminate the surface of the cathode and is then quickly accelerated by the high electric field. This process combined with a proper choice of solenoid field around, or immediately after, the gun, allows reaching very high beam brightness. These devices can find applications as injectors for free-electron lasers (FELs) [1-6], terahertz radiation generation [7,8], electron diffraction [9-13] and Compton sources [14].

Since the peak field at the cathode is directly proportional to the maximum achievable beam brightness, in the last generation of rf guns much effort has been spent, from the electromagnetic point of view, to find solutions to increase the field amplitude reducing the breakdown rate

Published by the American Physical Society under the terms of the Creative Commons Attribution 3.0 License. Further distribution of this work must maintain attribution to the author(s) and the published article's title, journal citation, and DOI. probability (BDR). To this purpose the goal was to find coupler geometries that reduce the pulsed heating on the surfaces [15] and irises profiles with lower peak surface electric field. Another important electromagnetic improvement has been the increase of the modal separation between the $\pi$ working mode and the so-called 0 mode. In fact, especially if the gun is fed using very short rf pulses, both modes are excited during the transient and the resulting beating can negatively affect the beam dynamics. The rf guns are, in general, realized by a brazing process of the machined parts. The brazing process requires a large vacuum furnace, is very expensive and poses a not negligible risk of failure. Furthermore, BDR studies in $\mathrm{X}$-band indicated that, avoiding the high temperature thermal stress of copper associated with brazing, one can reduce the BDR probability [15]. These motivations have been pointed out in novel gun design utilizing clamped parts [16].

In this paper we discuss the design and operation of a novel rf photoinjector built as a replacement of the SPARC_LAB injector which implements all these improvements and is fabricated using a new technique recently developed at LNF-INFN which avoids the brazing 
process. The current gun in operation at SPARC_LAB [17] uses a 1.6 cell standing wave structure of the the UCLA/ BNL/SLAC type [18] to obtain high beam brightness for the $150 \mathrm{MeV}$ SPARC_LAB accelerator in operation since 2004 at LNF-INFN [19]. The high brightness electron beams have been used for several experiments including SASE-FEL in visible light [20], demonstration of emittance preservation in velocity bunching [21] and FEL seeding experiments [22].

For the novel gun design discussed here, the iris profile has been designed with an elliptical shape and a large aperture to simultaneously reduce the peak surface electric field, increase the frequency separation between the two rf gun modes and increase the pumping speed on the half cell. The coupling window between the rectangular waveguide and the full cell has been strongly rounded to reduce the peak surface magnetic field and, as a consequence, the pulsed heating. Furthermore, the new SPARC gun has been realized without brazing using a novel process recently developed at LNF-INFN involving the use of special gaskets that guarantee (simultaneously) the vacuum seal and perfect rf contact when the structure is clamped.

In the first section of the paper we review the electromagnetic design criteria of the gun. In the second section we illustrate the fabrication process and the low power rf measurements results while, in the last section, we discuss the high power rf and beam test results performed at UCLA.

\section{ELECTROMAGNETIC DESIGN OF THE NEW GUN}

The electromagnetic design of the gun has been optimized using 2D and 3D electromagnetic codes. superfish [23] is used for the 2D design. The new 2D profile (compared with the previous gun profile) is given in Fig. 1(a). In the new gun the iris diameter $\left(2 r_{\text {iris }}\right)$ has been increased up to $36 \mathrm{~mm}$ (in the current gun it is $25 \mathrm{~mm}$ ). This allowed the mode separation to be $39 \mathrm{MHz}$ (that has to be compared with the $3.3 \mathrm{MHz}$ of the actual gun) also improving the pumping speed in the cathode region. We have also adopted an elliptical shape of the iris with a ratio 2 between the ellipse axes $(b / a)$. This allowed reducing the surface peak electric field on the iris itself with respect to the cathode field. Finally, also the beam pipe aperture $\left(2 r_{\text {pipe }}\right)$ has been increased to $32 \mathrm{~mm}$ allowing a better pumping of the gun full cell toward the pipe. The surface electric field normalized to the peak cathode field is given in Fig. 1(b). From the plot it is evident that the peak surface field has been reduced by as much as $15 \%$. The longitudinal electric field on axis is given in Fig. 1(c). The larger pipe aperture slightly increases the field penetration into the pipe itself. This has been verified to have a negligible effect on the beam dynamics. Also the half cell is slightly longer with respect to the previous device to optimize the beam dynamics performances.
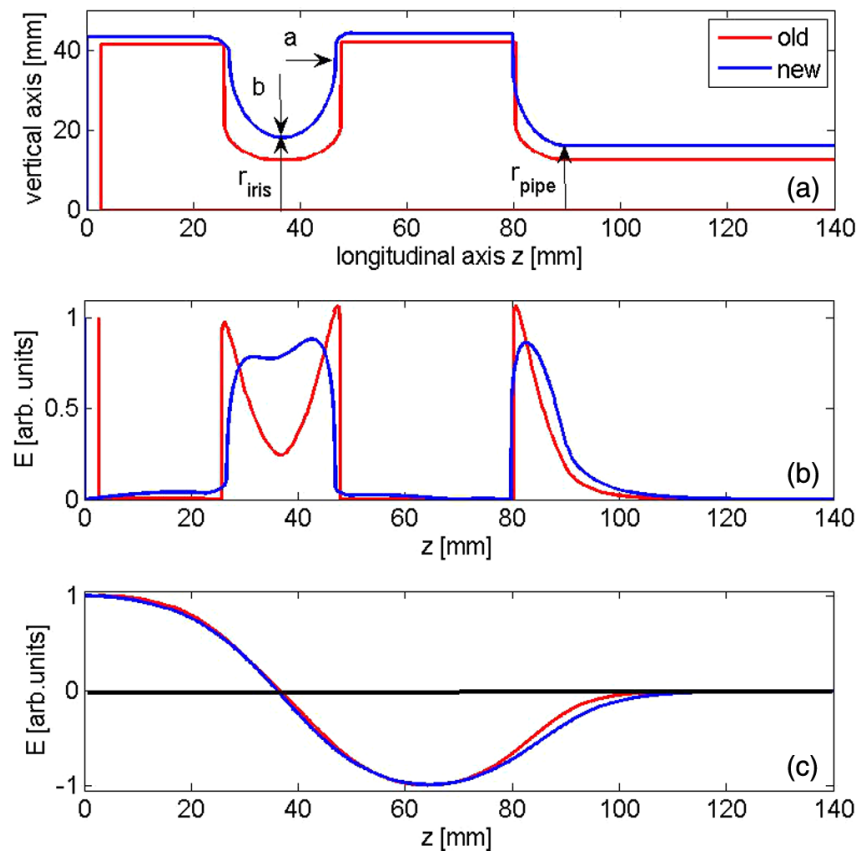

FIG. 1. (a) 2D profile (compared with the previous gun profile); (b) surface electric field normalized to the peak cathode field; (c) longitudinal electric field on axis.

Concerning the coupler design a strongly rounded coupler has been adopted. Its design has been completed with the 3D electromagnetic code HFSS [24]. Figure 2(a) shown the HFSS geometry of the gun while the surface peak magnetic field on the coupling hole (at $120 \mathrm{MV} / \mathrm{m}$ on the cathode) is given in Fig. 2(b). The maximum $\mathrm{H}$ field is $420 \mathrm{kA} / \mathrm{m}$ and the resulting pulsed heating with a $2 \mu \mathrm{s}$ rf pulse length is $<55^{\circ} \mathrm{C}$. This pulsed heating has been calculated according to the formula given in $[15,25]$.
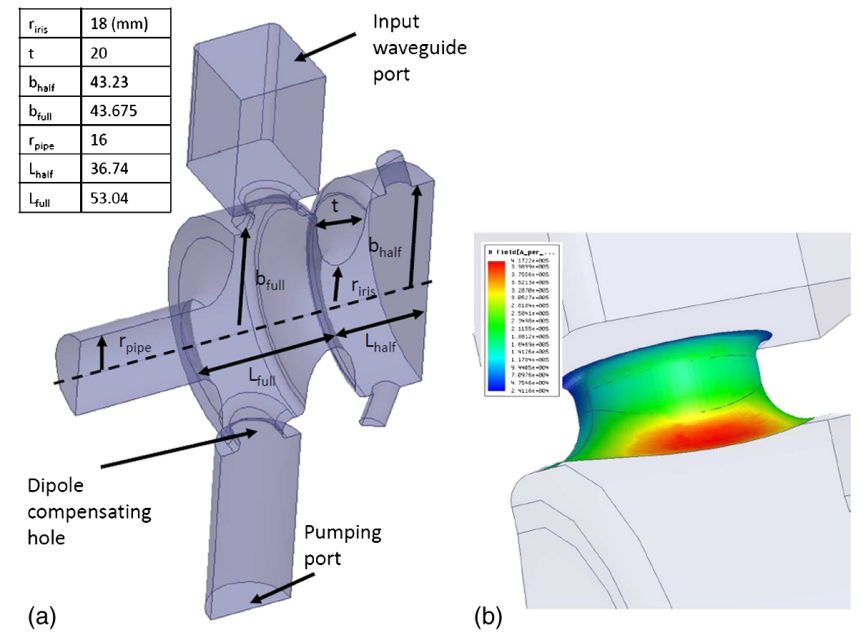

FIG. 2. (a) HFSS geometry of the rf gun; (b) detail of the surface peak magnetic field on the coupling hole at $120 \mathrm{MV} / \mathrm{m}$ on the cathode. 


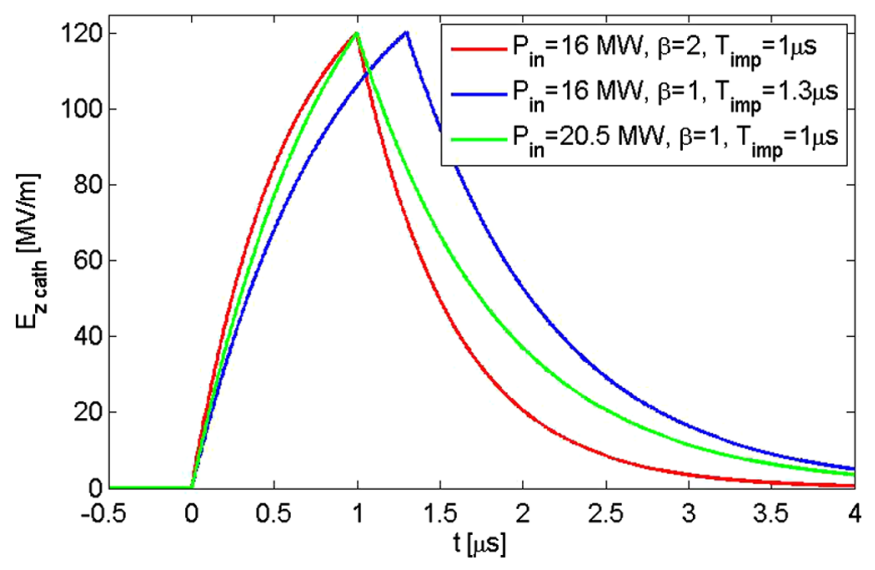

FIG. 3. Electric field on the cathode as a function of time in different cases.

The coupling coefficient $(\beta)$ has been increased in the new design from 1 to 2 . The corresponding lower loaded quality factor allows reaching the same cathode peak field with a shorter rf pulse length. Different cases are illustrated in Fig. 3 where the electric field on the cathode is plotted as a function of time for different $\beta$ coefficients and rf pulse length. A shorter rf pulse length (coupled with a higher input power) allows achieving, in principle, the same gradient with a reduction of the BDR. At the same time, when feeding the structure with a shorter rf pulse, if the frequency separation between the modes is not large enough, there is a residual field due to the 0 mode which is excited during the transient. For this reason, it is important in order to operate in this regime to strongly increase the mode frequency separation.

To compensate the dipole field component induced by the presence of the coupling hole, a symmetric port (connected to a circular pipe below cut off) has been inserted in the gun [26-28]. This port is also used as a pumping port. This "compensating" hole allows one to obtain nearly full cancellation of the on-axis dipole field. The residual magnetic field component is about $2.5 \times 10^{-4} \mathrm{~T}$ (at $120 \mathrm{MV} / \mathrm{m}$ cathode peak field). Concerning the quadrupole field component induced by the presence of the two holes, it has been evaluated according to the formulas given in [29] which are reported in the Appendix for completeness. The resulting integrated quadrupole gradient as a function of the injection phase is shown in Fig. 4. The result shows that the equivalent quadrupole gradient is small if compared to usual magnetic quadrupoles and beam dynamics simulations have confirmed a negligible effect on the beam quality. The final gun parameters are given in Table I.

With respect to previous fabricated guns like the LCLS gun [30] or the FERMI II gun [31], the new SPARC gun has a larger mode separation $(39 \mathrm{MHz}$ instead of about $15 \mathrm{MHz}$ of the two cited guns), a larger iris aperture and a rounded coupling slot instead of a $z$-coupling. The final

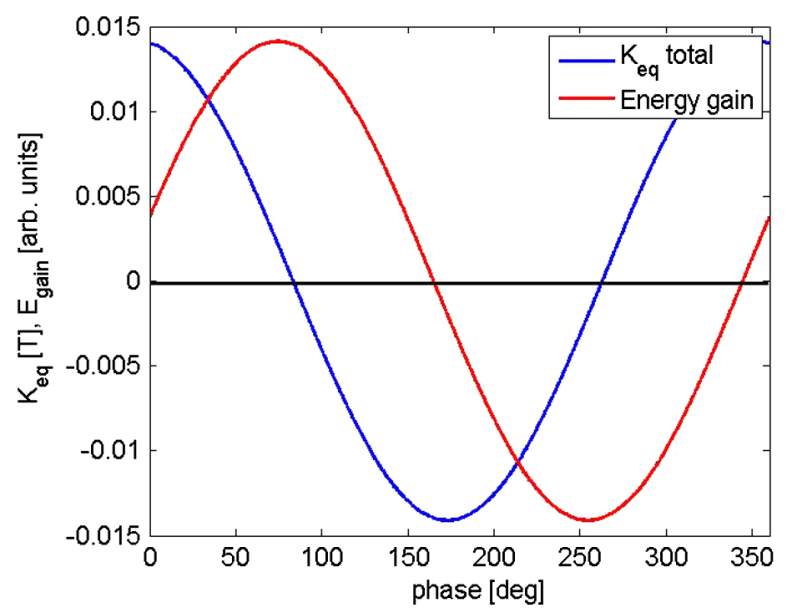

FIG. 4. Integrated quadrupole gradient as a function of the injection phase.

pulsed heating on the coupler hole is comparable with that of the LCLS and FERMI II guns. On the other hand, it is straightforward to understand that the $z$-coupling geometry cannot be adopted for clamped guns with gaskets, since the full cell is completely opened. In the LCLS and FERMI II guns the quadrupole component of the field is compensated by a race-track profile of the full cell while it is not compensated in this new SPARC gun. Nevertheless, as already pointed out, simulations taking into account this quadrupole field showed a negligible effect on beam emittance. An important advantage of this design choice is that the full cell of the SPARC gun can be machined by lathe because of the circular geometry while the LCLS and FERMI guns require a mill-based machining process.

Concerning the irises profile, we have adopted an elliptical shape also adopted in [31], which guarantees a

TABLE I. Radio frequency gun parameters theoretical (HFSS) and measured.

\begin{tabular}{|c|c|c|}
\hline Parameters & $\begin{array}{c}\text { Theoretical } \\
\text { (HFSS) }\end{array}$ & Measured \\
\hline$f_{\text {res }}$ & \multicolumn{2}{|c|}{$2.856[\mathrm{GHz}]$} \\
\hline$Q_{0}$ & 15300 & 15000 \\
\hline Coupling $\beta$ & 2 & 1.7 \\
\hline Shunt impedance $(\mathrm{R})$ & \multicolumn{2}{|c|}{$1.78[\mathrm{M} \Omega]$} \\
\hline$E_{\text {surf_peak }} / E_{\text {cathode }}$ & \multicolumn{2}{|c|}{0.85} \\
\hline$E_{\text {cathode }} / \sqrt{ }\left(P_{\text {diss }}\right)$ & \multicolumn{2}{|c|}{$38\left[\mathrm{MV} / \mathrm{m} / \mathrm{MW}^{0.5}\right]$} \\
\hline $\begin{array}{l}\text { Frequency separation between } \\
\text { the } 0 \text { and the } \pi \text {-mode }\end{array}$ & $39[\mathrm{MHz}]$ & $39.6[\mathrm{MHz}]$ \\
\hline$H_{\text {surf_peak }} @ E_{\text {cathode }}=120 \mathrm{MV} / \mathrm{m}$ & \multicolumn{2}{|c|}{$420[\mathrm{kA} / \mathrm{m}]$} \\
\hline $\begin{array}{l}\text { Pulsed heating ( } 2 \mu \mathrm{s} \text { rf pulse } \\
\text { length) }\end{array}$ & \multicolumn{2}{|c|}{$<55\left[{ }^{\circ} \mathrm{C}\right]$} \\
\hline Field flatness & $<1 \%$ & $<5 \%$ \\
\hline Repetition rate & \multicolumn{2}{|c|}{$10[\mathrm{~Hz}]$} \\
\hline Average dissipated power & \multicolumn{2}{|c|}{$75[\mathrm{~W}]$} \\
\hline Working temperature & \multicolumn{2}{|c|}{$20\left[{ }^{\circ} \mathrm{C}\right]$} \\
\hline
\end{tabular}


ratio between the cathode field and the irises one of about 0.85 . The laser ports edges have been rounded with a radius of curvature of $1 \mathrm{~mm}$ to maintain a pulsed heating below $50{ }^{\circ} \mathrm{C}$.

\section{FABRICATION PROCESS AND LOW POWER RF MEASUREMENTS}

The mechanical drawings of the gun are shown in Fig. 5. As already mentioned, the gun has been fabricated with a new technique recently developed at LNF that does not involve any brazing step. The body of the gun (part 1) has been fabricated from a single piece of Oxygen Free High Conductivity (OFHC) copper using diamond tools. The rounded coupler geometry has been realized with a five axis milling machine [32]. The full cell has then been sealed clamping the body of the gun with the closing cap (part 2) and using the special gasket that simultaneously guarantees the vacuum seal and the rf contact avoiding sharp edges and gaps. The waveguide has been fabricated separately and the rf contact and vacuum seal between the waveguide and the body of the gun has been obtained using another type gasket still developed at LNF-INFN. The device has been fabricated with a precision on the internal dimensions of $\pm 10 \mu \mathrm{m}$ while the surface roughness is maintained smaller than $200 \mathrm{~nm}$.

The detail of the gun assembly procedure is given in Fig. 6. In the figure we show the special rf-vacuum gaskets that have been used to simultaneously guarantee the vacuum tight and the rf contact. A sketch of the transverse profile of these special vacuum-rf gaskets is given in Fig. 7. A request of patent for this kind of gasket and for the realization of $\mathrm{rf}$ devices with them has been recently submitted [33]. The dimensions of the gasket are $1.3 \mathrm{~mm} \times$ $3 \mathrm{~mm}$ and the surface that guarantees the rf/vacuum contact is of $0.2 \mathrm{~mm}$. The gasket is compressed by only $0.15 \mathrm{~mm}$ in order to guarantee an elastic deformation of the gasket itself during gun assembly. The pumping port and the laser ports have been welded to the gun body. The cathode-to-body joint follows the same design as that adopted in previous fabricated guns [18].

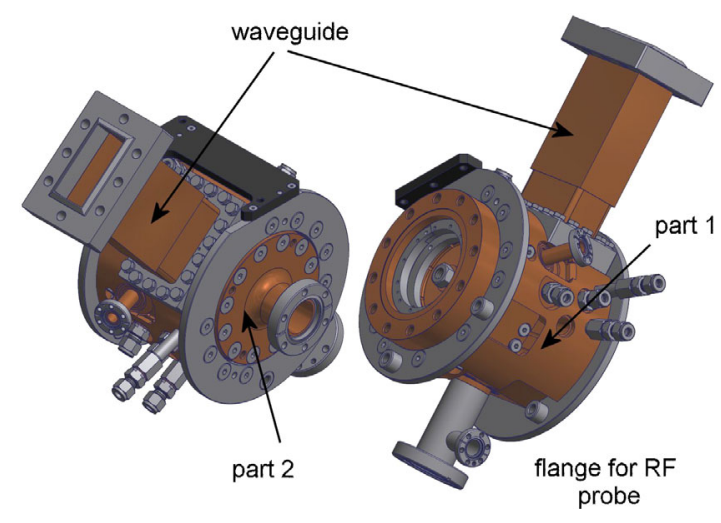

FIG. 5. Mechanical drawing of the gun.

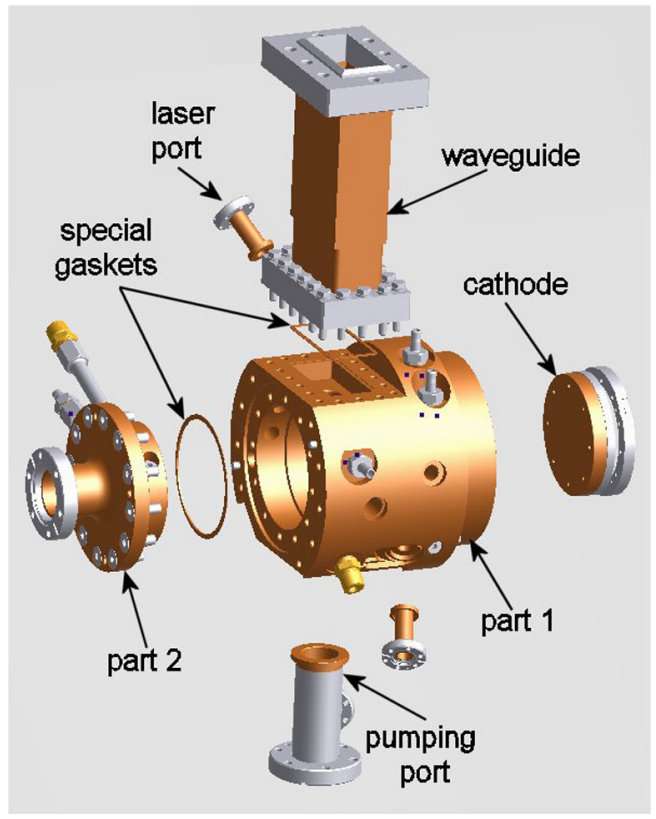

FIG. 6. Mechanical drawing of the gun: detail of the assembly procedure.

Before the final clamping, the gun has been cleaned with a detergent (ALMECO-19) and a mixture of organic (citric) acid and distilled water, in a bath with an ultrasound machine. After the assembly a bakeout at $150 \mathrm{deg}$ for 24 hours allowed reaching a vacuum pressure below $5 \times 10^{-10}$ mbar. Pictures of the assembled gun before and after the clamping are given in Fig. 8.

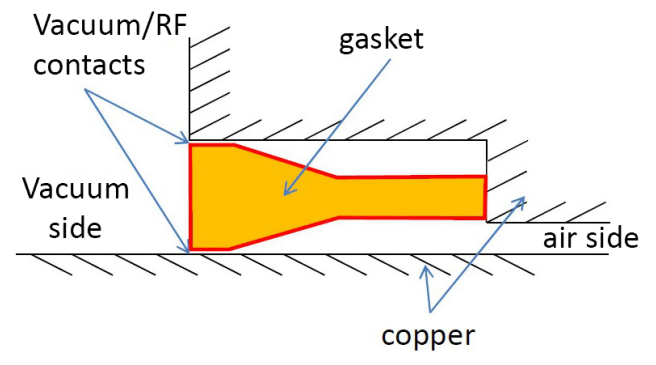

FIG. 7. Sketch of the transverse profile of the special vacuum/rf gasket used for gun clamping.
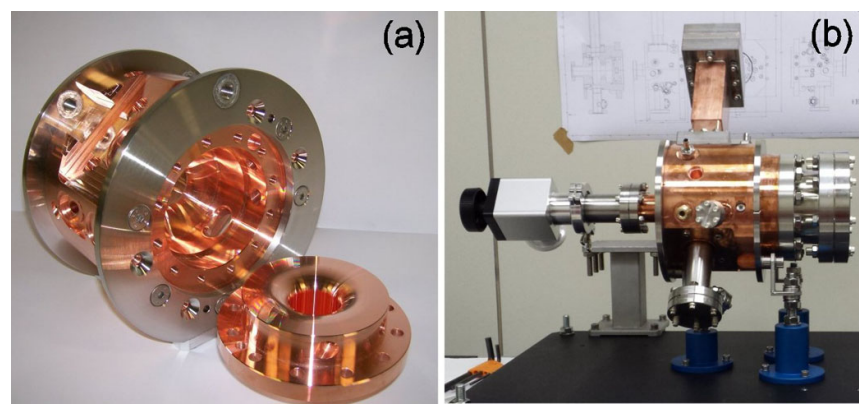

FIG. 8. Pictures of the gun before (a) and after the final assembly (b). 


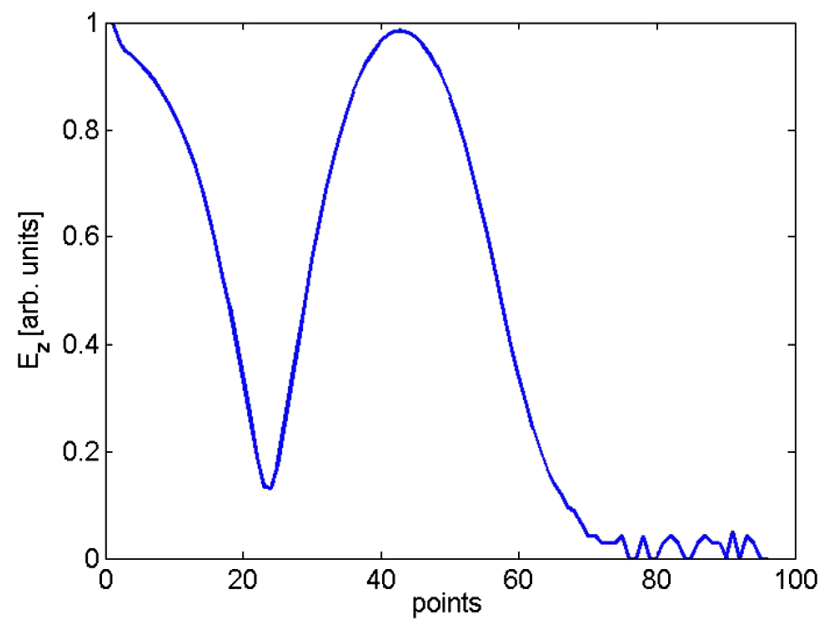

FIG. 9. Measured accelerating field profile after tuning.

Low power rf test and tuning of the gun have been performed using a network analyzer and the bead drop technique. The accelerating field profile after tuning and the reflection coefficient at the input port are given in Figs. 9 and 10 respectively. The resulting measured coupling coefficient is $\beta=1.7$ in fair agreement with the design value. The transmission coefficient between the input waveguide and the probe showing the two resonant modes of the gun are given in Fig. 11. The final rf gun parameters compared with the theoretical one are given in Table I.

\section{HIGH POWER TEST RESULTS}

High power rf tests have been performed at the University of California Los Angeles (UCLA) in the framework of a collaboration between INFN-LNF and UCLA. The gun has been installed in the Pegasus Laboratory and has been conditioned for about five weeks. The picture of the gun installed in the Pegasus Lab is given

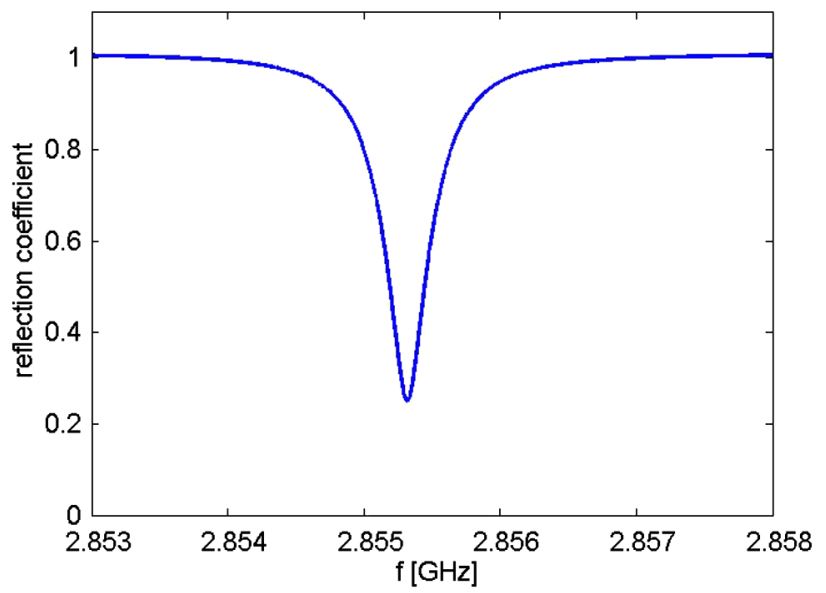

FIG. 10. Reflection coefficient at the input port (measured in air at $65 \%$ relative humidity and $20^{\circ} \mathrm{C}$ ).

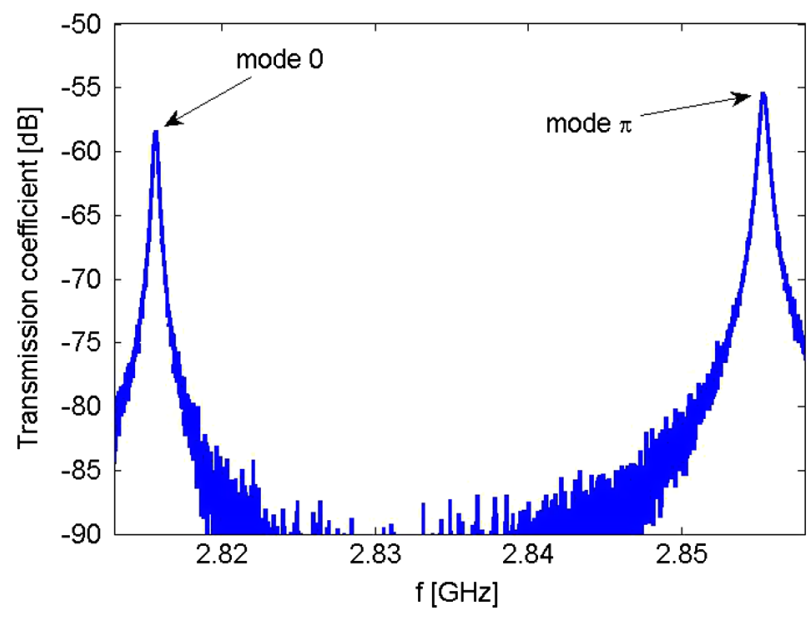

FIG. 11. Radio frequency gun resonant modes (measured in air at $60 \%$ relative humidity and $20^{\circ} \mathrm{C}$ ).

in Fig. 12. The gun was connected to three ion pumps: one ion pump of $30 \mathrm{l} / \mathrm{s}$ on the input waveguide, one of $100 \mathrm{l} / \mathrm{s}$ connected to the dummy hole in the full cell and one of $20 \mathrm{l} / \mathrm{s}$ connected to the back flange of the cathode. The structure has been fed by an XK5 klystron that was able to give a maximum power of $10 \mathrm{MW}$ with a maximum repetition rate of $5 \mathrm{~Hz}$ and a variable rf pulse length up to $2 \mu \mathrm{s}$. The conditioning has been done for about eight hours

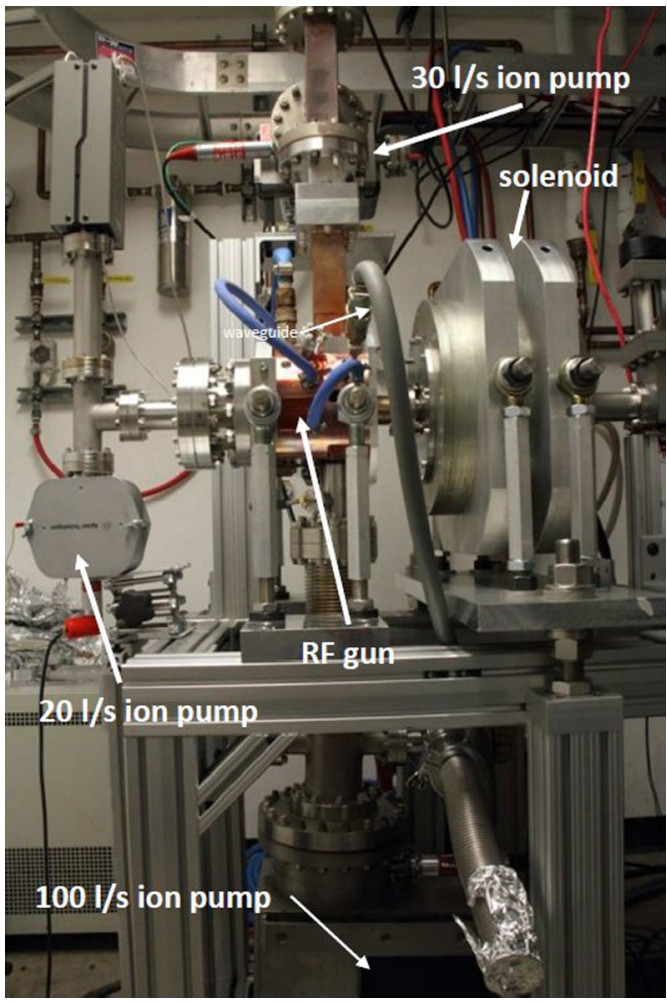

FIG. 12. Pictures of the rf gun installed in the Pegasus Laboratory at UCLA. 

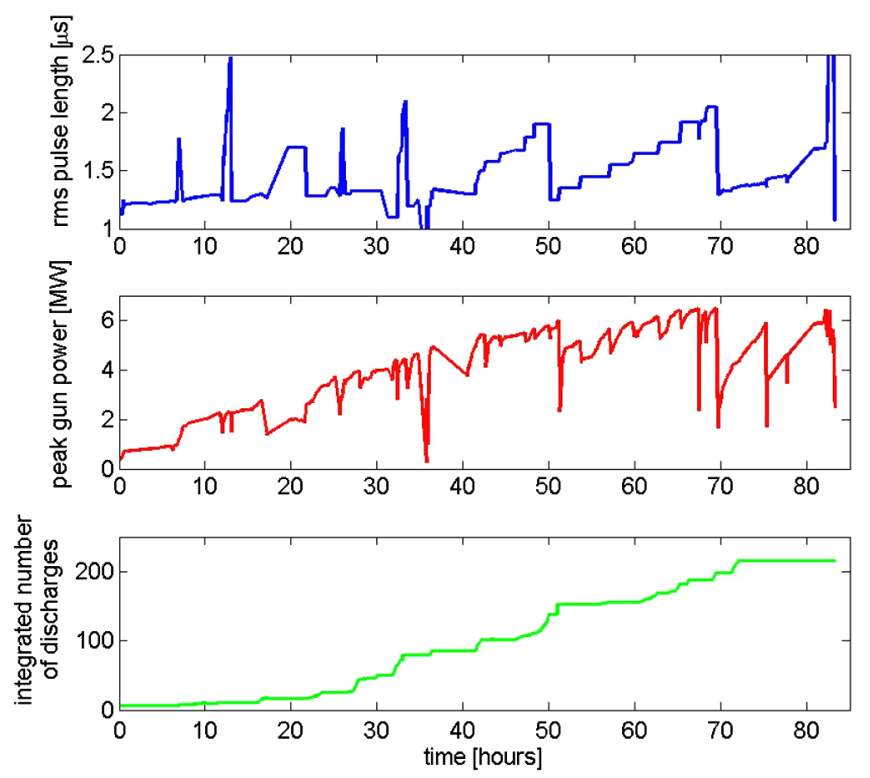

FIG. 13. Progress in power (measured from the gun probe), rms pulse length and integrated number of discharges during conditioning.

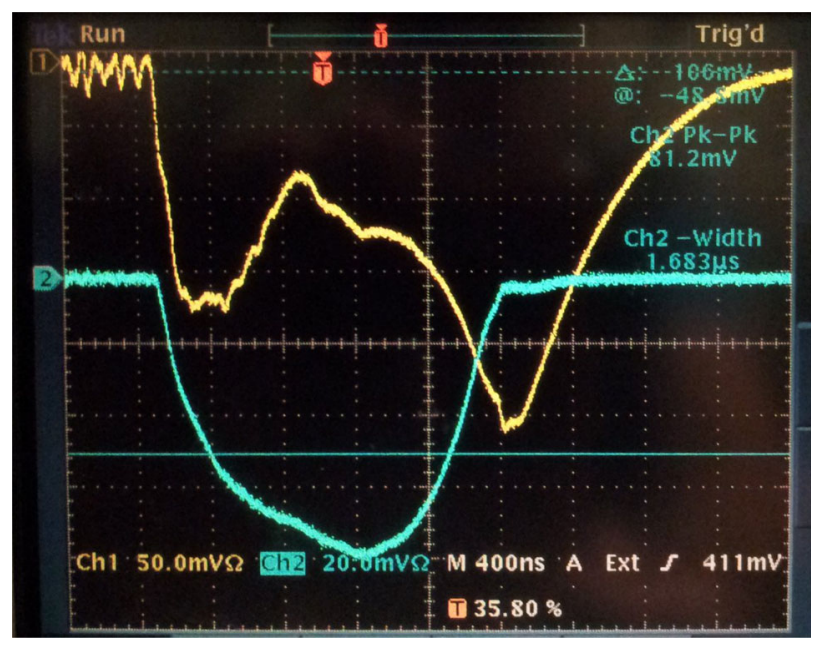

FIG. 14. Samples of monitoring signals during conditioning: cyan forward power, yellow reflected power. per day (working days) with a total amount of about 80 hours.

The power and the rf pulse length have been progressively increased starting with a short pulse length of $1 \mu \mathrm{s}$. Figure 13 shows the conditioning progress in terms of power dissipated into the gun (as measured through the probe) and the rms pulse length. During the conditioning we have monitored the current in the ion pumps and the $\mathrm{rf}$ signals (incident power, reflected power and transmitted power to the pickup). Samples of the incident and reflected input power are given in Fig. 14. In the first part of the conditioning (first week) the procedure was completely manual and the power were reduced by operators if a spike in vacuum due to a discharge occurred and/or a strong reflected power was measured. In the second part of the conditioning a semiautomatic procedure was indeed implemented and the power from the klystron was reduced if a strong variation on the pickup transmitted power was measured.

The gun reached about $8 \mathrm{MW}$ of maximum input power with an rms pulse length of $2 \mu \mathrm{s}$, corresponding to a $92 \mathrm{MV} / \mathrm{m}$ cathode peak field. The conditioning to higher fields was mainly limited by the available rf power. Moreover even if the rf gun has been designed to operate with very short rf pulses and high peak power, the klystron power profile had, indeed, a smooth initial filling time with an output peak power depending on the rf pulse length and it could reach power up to $10 \mathrm{MW}$ only for long pulse length $(>2.5 \mu \mathrm{s})$. Both these elements are not ideal when trying to push for high gradients. Moreover a full conditioning at the low repetition rate of $5 \mathrm{~Hz}$ requires a very long time to reach the final BDR. In the very short conditioning period we reached at the end a $\mathrm{BDR}<5 \times 10^{-6}$.

Electron beam has also been generated using a short (200 fs rms) UV laser pulse. The laser beam has been produced using a frequency tripled $\mathrm{Ti}: \mathrm{Sa}$ laser which illuminates the cathode at near normal incidence. The overall Pegasus experimental beam line is shown in Fig. 15. The electron beam energy as a function of $\mathrm{rf}$ phase has been measured for different power using a

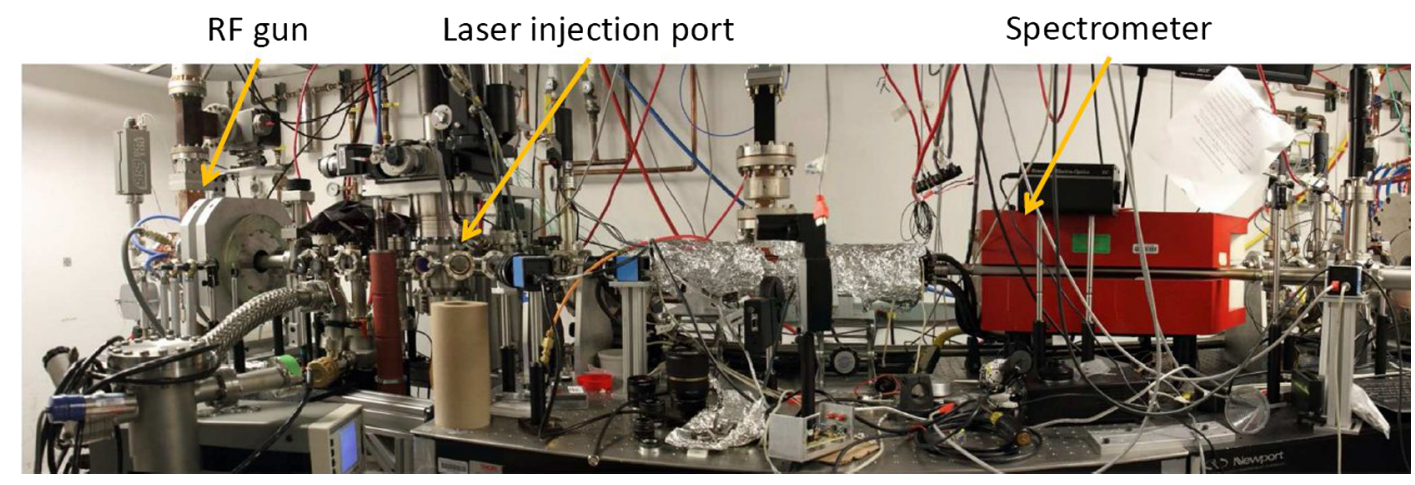

FIG. 15. Pegasus beam line. 
(a)

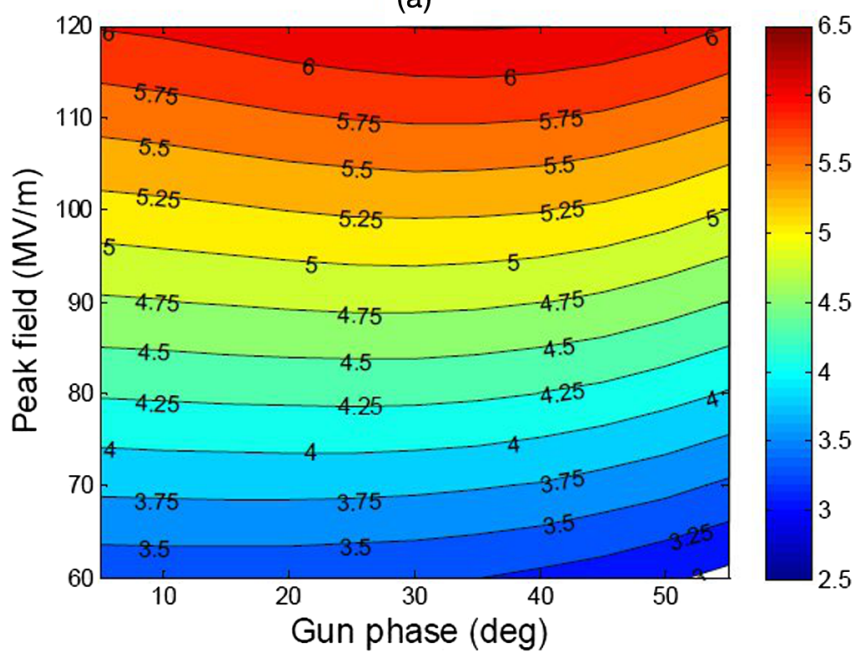

(b)

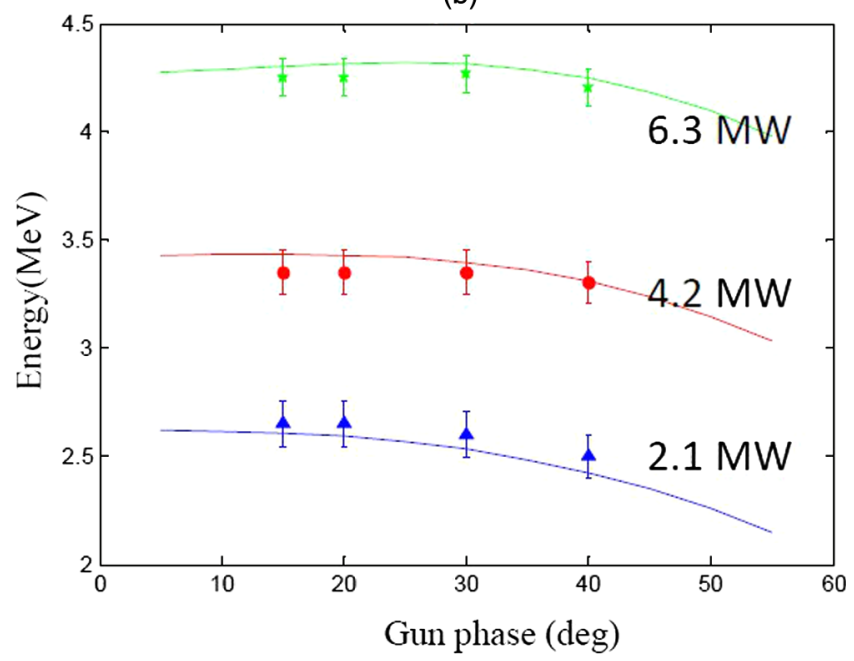

FIG. 16. (a) Theoretical maximum electron energy as a function of rf phase for different cathode peak field; (b) measured electron beam energy as a function of phase and for different power into the gun.

magnetic dipole spectrometer. The results are shown in Fig. 16 and are in good agreement with the gradients calculated by scaling the field with the dissipated power.

\section{CONCLUSIONS}

In the paper we discussed the design and operation of a novel rf photogun fabricated using a new technique recently developed at LNF-INFN which avoids the brazing process. The design implements also several features from the electromagnetic point of view such as high mode separation, low peak surface electric field at the iris and minimized pulsed heating on the coupler. Most of these features have been already applied in the LCLS and in the FERMI II guns which on the other hand necessitated a brazing step in the manufacturing process. Our novel fabrication design, avoiding brazing, strongly reduces the cost, realization time and the risk of failure for high gradient $\mathrm{rf}$ photoinjectors. The new rf gun has been built as a replacement of the SPARC_LAB injector and has been tested at high power at UCLA. The gun reached about $92 \mathrm{MV} / \mathrm{m}$ cathode peak field with an rms pulse length of $2 \mu \mathrm{s}$. First photoelectron beam was generated illuminating the cathode in the gun with a UV laser pulse. The results provided an independent confirmation of the peak gradient and demonstrate the use of this novel rf gun as a compact high gradient source for a high brightness photoinjector laboratory. Moreover the new clamping technique using vacuum/rf gaskets could be applied to other rf structures and other frequency bands opening new perspectives in the accelerator technology.

\section{ACKNOWLEDGMENTS}

The authors would like to thank A. Ghigo for his support in the project management. We would like also to thank P. Chimenti, R. Di Raddo, R. Clementi, M. Scampati,
S. Quaglia and the LINAC group at LNF for the technical support. P. M., E. P. and S. C. at UCLA were supported by NSF Award No. PHY-1415583.

\section{APPENDIX: MULTIPOLAR FIELD COMPONENTS INTRODUCED BY THE COUPLER}

Assuming that the gun has two perfectly symmetric holes (i.e., neglecting the fact that the gun is fed from just one of the two holes while the other is connected to a pipe below cut off) the azimuthal magnetic field can be developed as follows:

$$
B_{\phi}(r, \phi, z) \cong A_{o}(z) r+\sum_{n=1}^{\infty} A_{2 n}(z) \cos (2 n \phi) r^{2 n-1},
$$

where the $A_{2 \mathrm{n}}$ components depend on the longitudinal coordinate $z$.

The quadrupolar component is the component associated to the term with $n=1$ and the gradient of the quadrupole component is exactly the term $A_{2}$.

Figure 17 illustrates the azimuthal magnetic field in the center of the full cell for three different circle radii. The plot is referred to the case of a $120 \mathrm{MV} / \mathrm{m}$ accelerating field on the cathode. The field profile is mainly driven by the quadrupolar component ( $2 \varphi$ dependence).

In principle it is possible to calculate all multipolar components at different longitudinal coordinates by calculating the Fourier series components in different arcs. As a first order we have estimated the quadrupole gradient along the structure in the following way:

$$
A_{2}(z) \cong \frac{B_{\max }\left(r_{0}, z\right)-B_{\min }\left(r_{0}, z\right)}{2 r_{0}},
$$




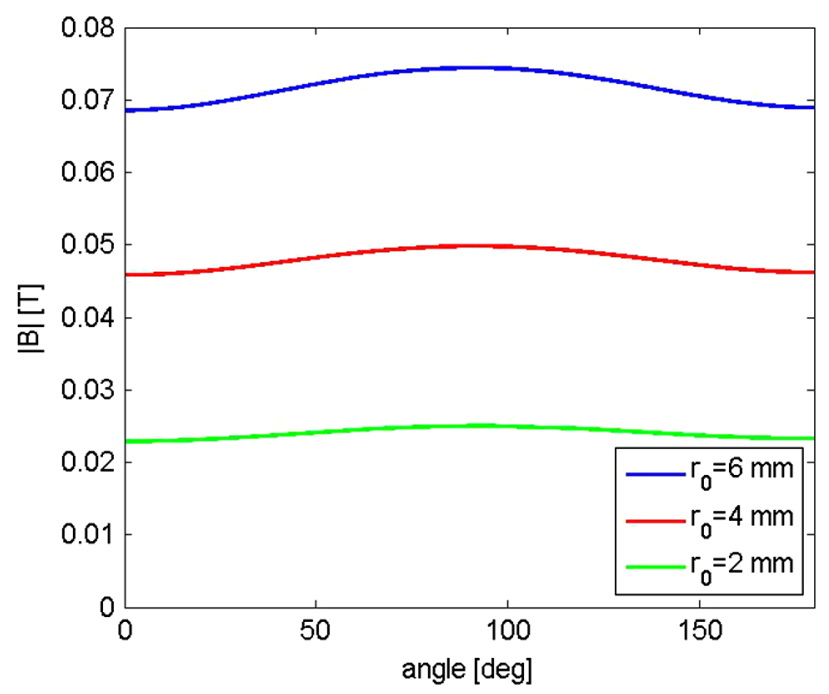

FIG. 17. Azimuthal magnetic field in the center of the full cell (referred to the case of a $120 \mathrm{MV} / \mathrm{m}$ accelerating field on the cathode) for three different circle radii.

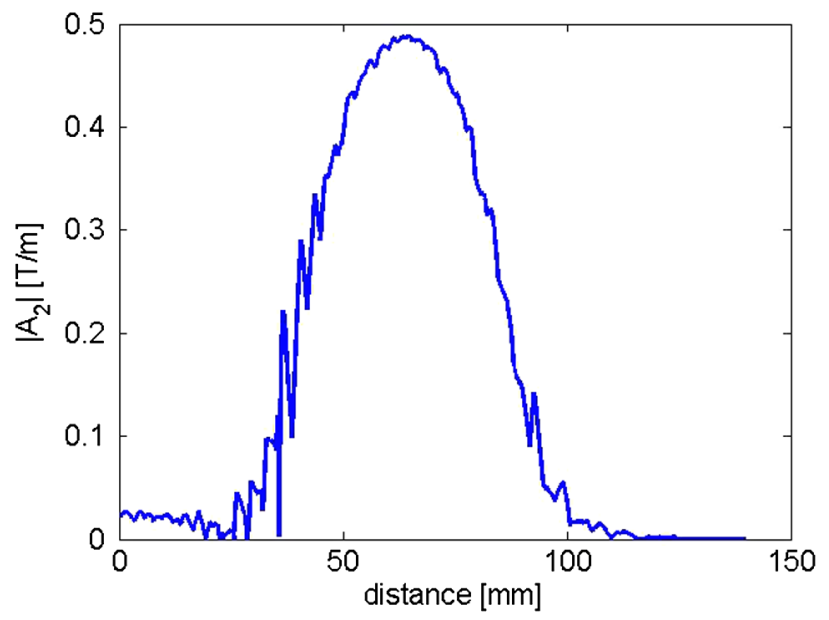

FIG. 18. Absolute value of the quadrupole gradient calculated using Eq. (A1) for $r_{0}=8 \mathrm{~mm}$.

where $B_{\max }$ and $B_{\min }$ are the maximum and minimum of the magnetic field at a certain distance $r_{0}$ from the axis and at a given longitudinal coordinate $z$. Figure 18 shows the absolute value of the quadrupole gradient for $r=8 \mathrm{~mm}$. The integrated quadrupole gradient as a function of the injection phase is shown in Fig. 4 (text of the paper).

[1] P. G. O'Shea et al., in Proceedings of the 1991 Particle Accelerator Conference, San Francisco, CA, 1991 (IEEE, New York, 1991), p. 2754.

[2] D. H. Dowell et al., Appl. Phys. Lett. 63, 2035 (1993).

[3] R. Dei-Cas et al., Nucl. Instrum. Methods Phys. Res., Sect. A 296, 209 (1990).
[4] S. Schreiber, in Proceedings of the European Particle Accelerator Conference, Vienna, 2000 (EPS, Geneva, 2000), p. 309.

[5] R. Akre et al., Phys. Rev. ST Accel. Beams 11, 030703 (2008).

[6] H. S. Kang and S. H. Nam, in Proceedings of the 32nd Free Electron Laser Conference, Malmö, Sweden (Max-lab, Sweden, 2010), p. 155.

[7] R. Kuroda, Nucl. Instrum. Methods Phys. Res., Sect. A 593, 91 (2008).

[8] C. Yim et al., in Proceedings of the International Particle Accelerator Conference, Kyoto, Japan (ICR, Kyoto, 2010), p. 1059.

[9] J. B. Hasting, F. M. Rudakov, D. H. Dowell, J. F. Schmerge, J. D. Cardoza, J. M. Castro, S. M. Gierman, H. Loos, and P. M. Weber, Appl. Phys. Lett. 89, 184109 (2006).

[10] R. Li, C. Tang, Y. Du, W. Huang, Q. Du, J. Shi, L. Yan, and X. Wang, Rev. Sci. Instrum. 80, 083303 (2009).

[11] J. Yang, K. Kan, N. Naruse, Y. Yoshida, K. Tanimura, and J. Urakawa, Radiat. Phys. Chem. 78, 1106 (2009).

[12] P. Musumeci, J. T. Moody, C. M. Scoby, M. S. Gutierrez, H. A. Bender, and N. S. Wilcox, Rev. Sci. Instrum. 81, 013306 (2010).

[13] J.-H. Han, Phys. Rev. ST Accel. Beams 14, 050101 (2011).

[14] R. Kuroda, H. Toyokawa, M. Yasumoto, H. IkeuraSekiguchi, M. Koike, K. Yamada, T. Yanagida, T. Nakajyo, F Sakai, and K. Mori, Nucl. Instrum. Methods Phys. Res., Sect. A 637, S183 (2011).

[15] V. A. Dolgashev et al., RF breakdown in normal conducting single-cell structures, in Proceedings of the 21st Particle Accelerator Conference, Knoxville, TN, 2005 (IEEE, Piscataway, NJ, 2005).

[16] S. B. van der Geer et al., Design, Construction and Operation of the Dutch RF-Photoguns, in Proceedings of the 23rd Particle Accelerator Conference, Vancouver, Canada, 2009 (IEEE, Piscataway, NJ, 2009), MO6RFP086.

[17] http://www.lnf.infn.it/acceleratori/sparc_lab/.

[18] D. Palmer, Ph.D. thesis, The next generation photoinjector, Stanford Linear Accelerator Center, Stanford University, 1998.

[19] D. Alesini et al., Nucl. Instrum. Methods Phys. Res., Sect. A 507, 345 (2003).

[20] M. Ferrario et al., in Proceedings of the 23rd Particle Accelerator Conference, Vancouver, Canada, 2009 (Ref. [16]).

[21] M. Ferrario et al., Phys. Rev. Lett. 104, 054801 (2010).

[22] M. Labat et al., Phys. Rev. Lett. 107, 224801 (2011).

[23] http://laacg.lanl.gov/laacg/services/download_sf.phtml.

[24] http://www.ansys.com.

[25] D. Alesini, arXiv:1112.3201.

[26] D. T. Palmer, R. H. Miller, and H. Winick, Microwave measurements of the BNL/SLAC/UCLA 1.6 cell photocathode rf gun, in Proceedings of the Particle Accelerator Conference, Dallas, TX, 1995 (IEEE, New York, 1995), p. 982.

[27] X. Guan, C. Tang, H. Ghen, W. Huang, X. He, P. Xu, and R. Li, Nucl. Instrum. Methods Phys. Res., Sect. A 574, 17 (2007).

[28] M. S. Chae, J. H. Hong, Y. W. Parc, In Soo Ko, S. J. Park, H. J. Qian, W. H. Huang, and C. X. Tang, Phys. Rev. ST Accel. Beams 14, 104203 (2011). 
[29] D. Alesini et al., J. Instrum. 8, P05004 (2013).

[30] C. Limborg et al., RF design of the LCLS gun, Report No. LCLS-TN-05-3, 2005.

[31] L. Faillace et al., Status of the Fermi II rf gun at Sincrotrone Trieste, in Proceedings of LINAC2014, Geneva, Switzerland (Jacow, Geneva, 2014).
[32] http://www.comeb.it.

[33] D. Alesini, V. Lollo, and A. Battisti, Procedimento per la realizzazione di una guarnizione metallica da vuoto e a radiofrequenza e struttura che la incorpora, Italian patent Application No. 102015000008811 (2015). 